\title{
A Joint Encryption and Digital Image Watermarking System using WBC Transform in Tele Radiology
}

\author{
Kullayamma \\ SVUCE, Department of ECE, Tirupati, INDIA \\ Sathyanarayana \\ AITS, Department of ECE, Tirupati, INDIA
}

\begin{abstract}
Tele-radiology is an emerging field in the stream of medical diagnosis in which maintenance and transmission of the medical reports like MRI, CT-Scan and X-Ray can be done through internet. The main objective of Teleradiology is automated report handling of the patients and accessing the report by proper authorization. The issue arises at verification, validation of the medical images sent through the internet. To address the issue of verification, in this paper we have used a secret key image with bit-x-or encryption methodology, first a key image of the physician is taken, as a reference and a secret key is generated by using bit-X-OR operation which is further used as original key for encrypting the watermarked image. The validation of medical images authenticates the patient image to whom the particular report belongs and this process is addressed by using watermarking methodology implemented through wavelet based counter let transform (WBC). The encryption of the image is done post to the watermarking. The efficiency of watermarking methodology is verified with PSNR, MSE and Correlation Coefficient, significant improvement is observed in proposed methodology.
\end{abstract}

Keywords: Bit -X-OR operation, Encryption, Key image, Secret key ,. Tele-radiology, Wavelet based contour let Transform (WBC).

\section{INTRODUCTION}

Evolution of globalization and usage of internet has emerged an E-Health Management System in which the diagnosis and analysis of the treatment of the diseases is done through the internet. Tele-radiology is one among the hierarchy of the E-Health Management System. The process of Tele-radiology involves in sending and receiving the medical images like X-Ray, MRI, and CT scan through a separate mail management system in the internet [1]. Here in the process of sending images may undergone fraud tampering, unauthenticated usage [1] of image without any right. To get rid of this problem in this paper we proposed a secret key encryption methodology for verification of valid user and validation of the patient data is achieved through watermarking scheme introduced in the medical image. Secure encryption methodology is proposed in this paper first an authority image that is taken as a reference image then that reference image water image format is extracted, by bit-x-or operation of both reference and water image. We generated a secret key which is further used as key for encrypting and decrypting the watermarked image.

Watermarking the patient image into the Biomedical image is an efficient mechanism that handles the valid report carrying of the patient and validates the patient image before analyzing their medical image. In the process of watermarking, decomposition of the image to get an appropriate sub-band is an important procedure since decomposition, selecting appropriate sub-band and inserting the patient details into the sub band should not affect the visual information of the original image, so we addressed above problem using wavelet based contour let transform (WBC). Prior to this method wavelet and contour let transforms were used which are not capable to handle the 2-D singularities of the image and having redundancy factor of $4 / 3$ respectively, so used WBC in which wavelet transform is applied first then contour let transform is applied to achieve the quad tree decomposition of both low pass and high pass channels. The PSNR, MSE and Correlation coefficient (CC) are compared for respective decomposition methodologies as shown in the Table I.

\section{SECRET KEY ENCRYPTION (BIT-X-OR OPERATION)}


In the field of Tele-radiology providing the security for the digital bio-medical imaging is a crucial issue. The transmitted bio-medical image in the internet should ensure the security and privacy of the particular patient to whom that report belongs. To prevent the un-authorized access of the bio-medical image a simple and powerful image encryption methodology is proposed by using bit-x-or operation. As this methodology comes under the hierarchy of the symmetrical key format where both encryption and decryption of the image uses same key.

There were various algorithms for encryption like AES,DES and RSA with high security but all are efficient for text data not for images and implementation of algorithm is also complex, so a simple and secure encryption image based methodology is implemented. It is named as simple because as simple bit-x -or operation is performed between the images, which is done at both encryption and decryption stages. To increase the security level a new key image called secret key is generated by using original key image of the physician and water image of the physician at both encryption and decryption stages.

The following algorithm shows how the encryption mechanism is implemented.

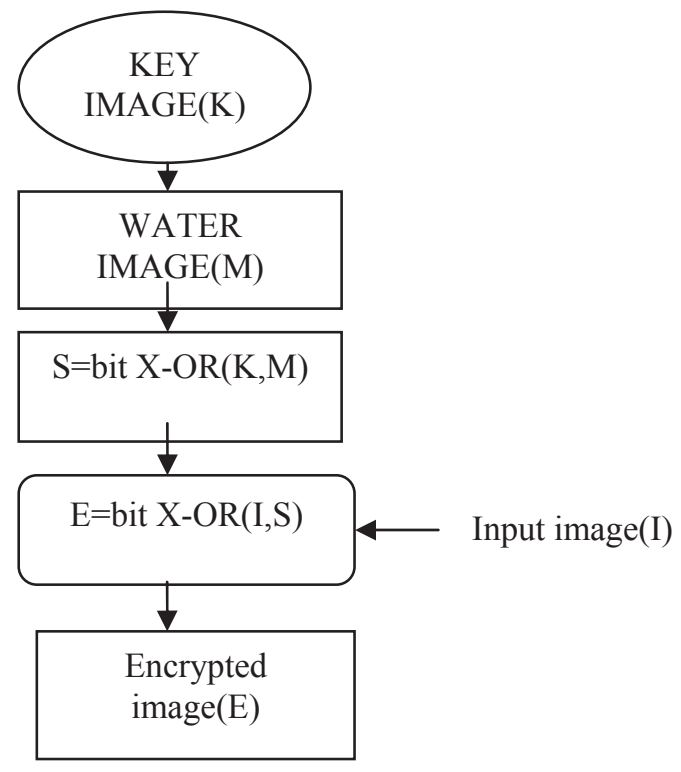

In the above algorithm $\mathrm{S}$ indicates the secret key which is obtained by performing the bit-xor operation of the original key (k) and water image version of the original key Image(M).

The following algorithm shows how the decryption mechanism is implemented 


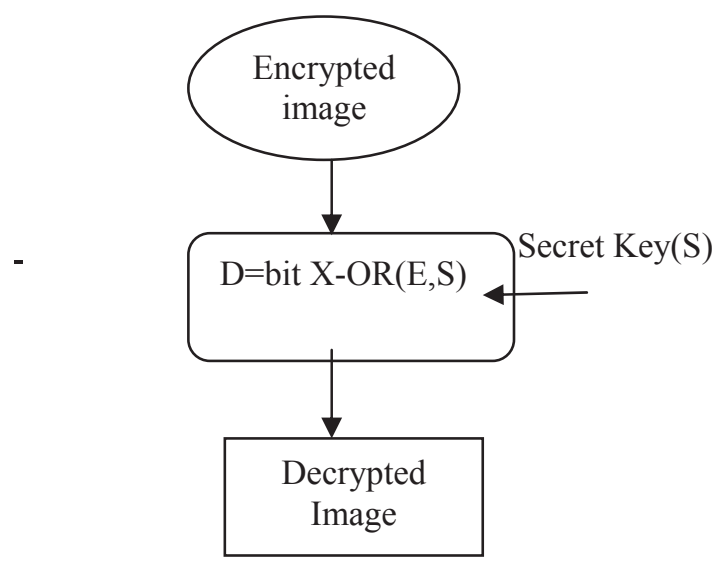

The schematic depicts how the decryption mechanism is implemented using the secret key(S) and the Encrypted image(E) to get the decrypted image(D).

\section{WAVELET BASED CONTOUR LET TRANSFORM (WBC)}

The validation of patient details is addressed by watermarking. In the process of watermarking, decomposition of image into sub-bands is an important procedure which is implemented by applying WBC transform. Prior to WBC transform there were two widely used transforms one is Harr-Wavelet and other is contour let based. The HarrWavelet has limitations in finding decomposition levels and 2-D Singularities of the image, to alleviate these limitations Contour let Transform was used which is an efficient transform in representing the two dimensional signals, capturing the intrinsic geometric structure of image, and also provides multi-resolution and directional image expansion[6]. It is derived using pyramidal directional filter banks which is a combination of laplacian pyramid and directional filter bank the laplacian pyramid has limitation in redundancy, about a factor of 4/3[7]. So we used a finest technique to decompose the image that is WBC transform. It is analogous to Contour let transform but the first stage laplacian pyramid filter bank is replaced with wavelet transform implemented through separable filter bank the second stage of WBC is a directional filter bank DFB provides angular decomposition implemented through non-separable filter banks.

The implementation of WBC [7]involves each level (M) in the wavelet transform, we obtain the traditional three high pass bands corresponding to the LH, HL, and $\mathrm{HH}$ bands.

We apply DFB with the same number of directions to each band in a given level (m). Starting from the desired maximum number of directions $\mathrm{N}_{\mathrm{D}}=2^{\mathrm{L}}$ on the finest level of the wavelet transform $\mathrm{M}$, we decrease the number of directions at every other dyadic scale when we proceed through the coarser levels $(\mathrm{m}<\mathrm{M})$. This way, we could achieve the anisotropy scaling law. Which is the advantage of WBCT over the Contour let transform. Quad-tree decomposition of both low pass and high pass channels in wavelets and apply the DFB on each sub-band as shown in the above figure
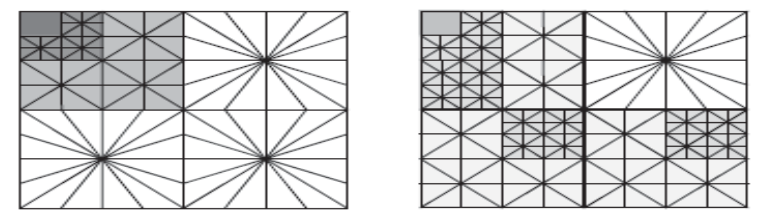

Fig 1: WBCT using 3 dyadic wavelet levels $(\mathrm{N}=8)$ and wavelet based contour let packets Following a similar procedure outlined in [3], for an $l$ level DFB we have $2^{l}$ directional sub bands with $\mathrm{G}_{\mathrm{K}}{ }^{(1)}, 0<\mathrm{K}<2^{\mathrm{L}}$ equivalent synthesis filters and the overall down sampling matrices of $\mathrm{S}_{\mathrm{K}}{ }^{(1)}$

\section{SYSTEM ARCHITECTURE}

The System Architecture involves following steps for processing the Bio-medical image 
1. Load the Bio-Medical Image to the system and apply contour let transform for finding the edges of the image.

2. Apply Harr Wavelet for decomposition of the image and further quad-tree decomposition is done through passing the sub-band to contour let transform .

3. Embedding the patient image into the appropriate sub-band is done by watermarking scheme using wavelet based contour let transform to obtain watermarked image.

4. Select an appropriate physician image for verification purpose and generate a secret key by bit-xor operating the Physician image and it's water image version.

5. Encrypt the watermarked host image with the secret key with bit-xor operation.

6. Retrieval of the watermarked patient image from the bio-medical image is done by decrypting the watermarked image with secret key.

7. If decryption of the watermarked image is done with appropriate secret key then water mark image of valid patient is extracted from the bio-medical image.

8. If secret key is not appropriate, the patient image is not extracted from the bio-medical image.

Schematic of cited system Architecture can be viewed below.

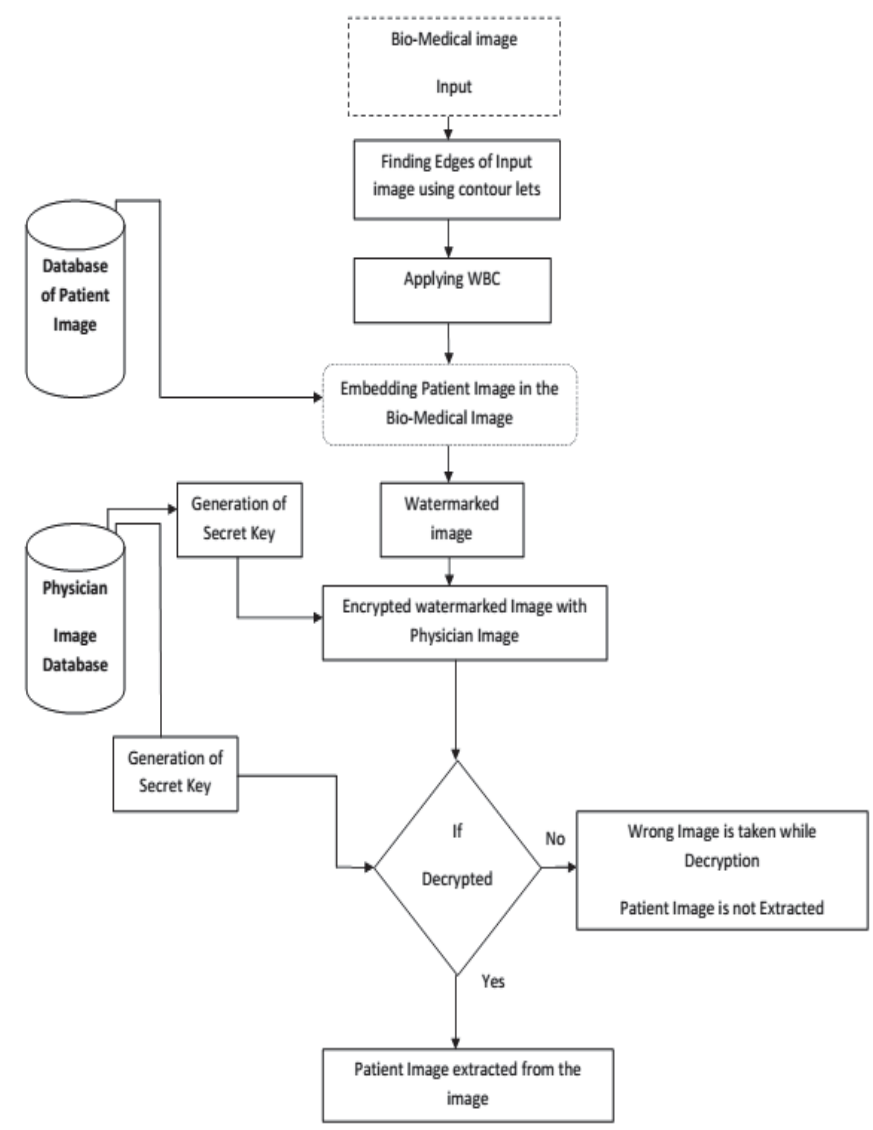




\section{RESULTS AND DISCUSSIONS}

The Host Bio-medical image of T-Cross section area of a brain with a tumor is loaded to the system for displaying the input image and applying the contour let transform that identifies the edges of the cross sectional image. The schematic of input image and it's edges are as shown in below figure.

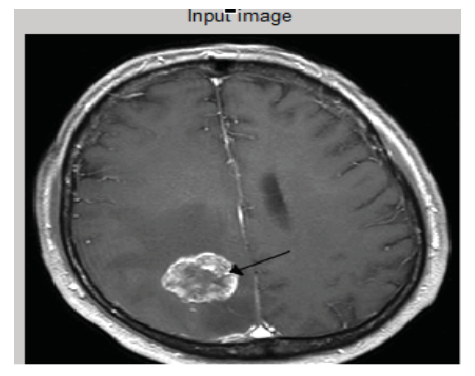

Fig 2: Input Image

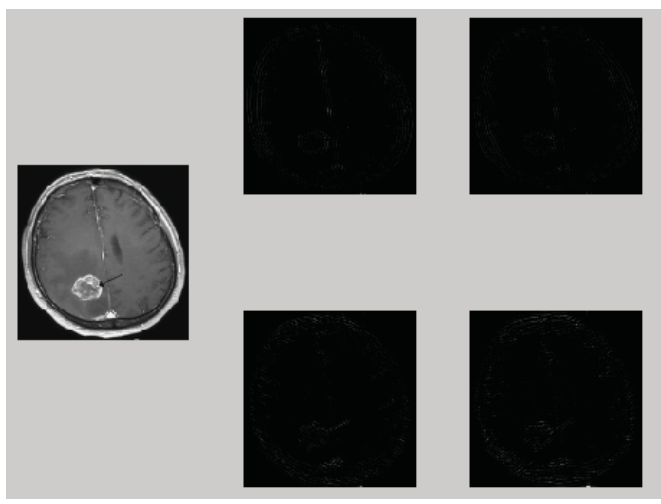

Fig 3: Edges of input image

wavelet based contour let transform has been implemented by using Harr and Pyramidal Directional Filter Bank to select appropriate sub band for watermarking the patient details into the image. The WBC of given image are as shown figure.

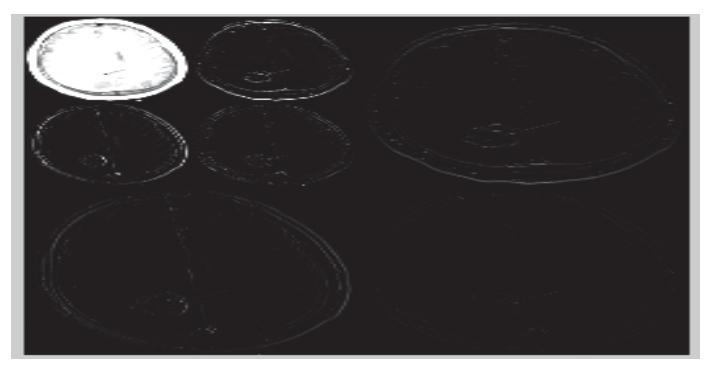

Fig 4: Wavelet Based Contour let

The next step is to watermark the patient image into the sub-band of the host image which undergone WBC. The watermark image and watermarked host image of the inserted patient image. 


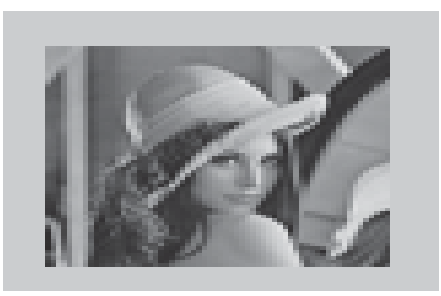

Fig 5: Watermark Patient image

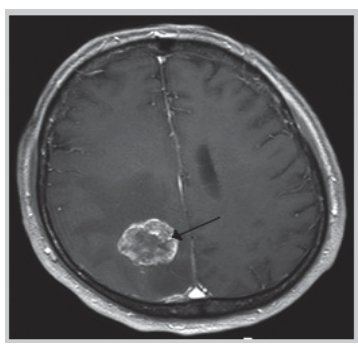

Fig 6: Water marked Host Image with patient image

A key image of authorized physician image is collected from a database and a secret key is generated for encrypting the watermarked image with the secret key the key image(physician image) and secret key image are depicted in fig 7 and fig 8 .

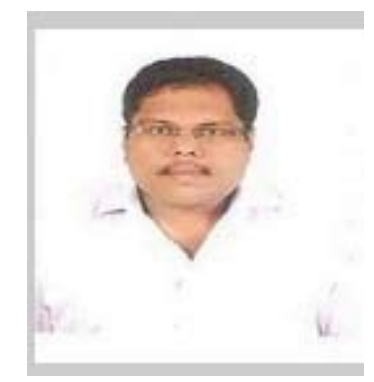

Fig7 Key image of physician

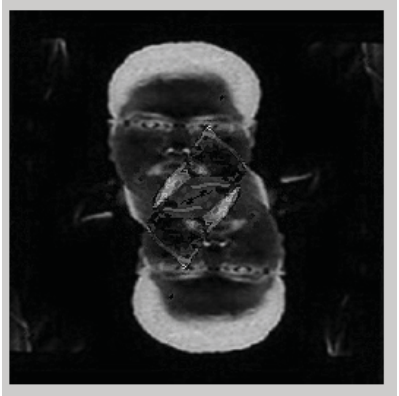

Fig 8: Secret key image

The secret key image is used for encrypting the watermarked image and the encrypted watermarked image is depicted below 


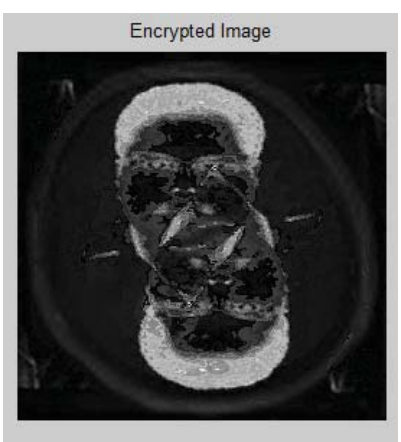

Fig.9: Encrypted watermarked image

If the decryption the water marked image is done with valid secret key generation of authorized physician image the watermark of the patient image is extracted as shown in the fig 10

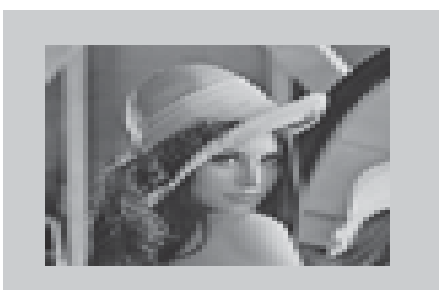

Fig 10: extracted watermark

If the decryption is not valid then watermark of the patient image is not extracted a message is displayed as shown in the figure 11

you have entered the wrong key while decryption

fig 11 wrong key of decryption message

The same process is recurred to the various input bio medical images which taken from the data set as shown in the figure $\mathrm{a}$, figure $\mathrm{b}$ and figure $\mathrm{c}$.

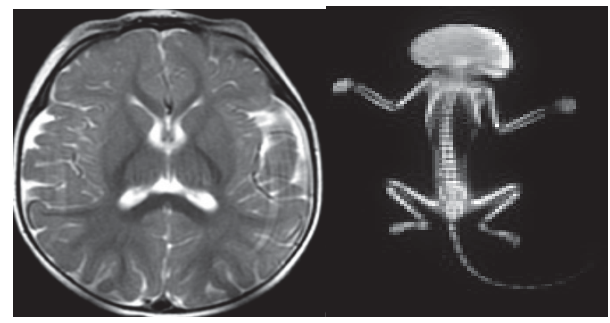

Fig a: Input Image 2 Fig b: Input Image3

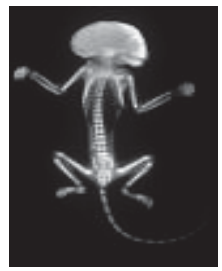

Fig c: Input Image 3

The Efficiency of watermarking methodologies is tested for various input images and water marked images using PSNR MSE and Correlation Coefficient(CC) the various input images are as shown in the figure 2, figure $a$, figure $b$ and figure $\mathrm{c}$. The obtained results are depicted in Table I. 
Table I Comparison of efficiency of watermarked images using wavelet, contour let and WBC Transforms.

\begin{tabular}{|c|c|c|c|c|c|c|c|c|c|}
\hline FIGURES & \multicolumn{3}{|c|}{ WAVELET TRANSFORM } & \multicolumn{3}{|c|}{ CONTOUR LET TRANSFORM } & \multicolumn{3}{|c|}{ WBC TRANSFORM } \\
\hline & PSNR & MSE & $\begin{array}{l}\text { CORRELATION } \\
\text { COEFFICIENT }\end{array}$ & PSNR & MSE & $\begin{array}{l}\text { CORRELATION } \\
\text { COEFFICIENT }\end{array}$ & PSNR & MSE & $\begin{array}{l}\text { CORRELATION } \\
\text { COEFFICIENT }\end{array}$ \\
\hline Fig: 2 & 39.6668 & 0.0189 & 0.5380 & 64.3186 & 13.9201 & 0.9179 & 64.7848 & 99.8876 & 1.0000 \\
\hline Fig: a & 24.9935 & 0.0821 & 0.7313 & 60.1408 & 31.7636 & 0.9236 & 63.9735 & 100 & 1.0000 \\
\hline Fig: b & 35.8651 & 0.0277 & 0.6720 & 55.5877 & 7.1251 & 0.8867 & 64.9463 & 98.2873 & 0.9998 \\
\hline Fig: c & 28.9199 & 0.0555 & 0.4715 & 56.1125 & 5.3523 & 0.9345 & 64.7817 & 99.9183 & 1.0000 \\
\hline
\end{tabular}

\section{CONCLUSION}

Verification and validation of Bio-medical image has been achieved through this proposed system. The encryption of the watermarked image ensures the verification of valid authorized physician can access the biomedical image through decrypting the watermarked image. we also used WBC transform, one of the finest transform for water marking the patient image into the image. It ensures the visual information of the Bio-medical image is not altered. we also compared efficiency of the proposed decomposition methodology for watermarking, with existing methods (i.e.) using wavelet and contour let transforms for decompositions and observed significant improvement in all the metrics like PSNR,MSE and Correlation coefficient(CC) in proposed watermarking methodology using WBC. Further the proposed system can be extended to bio-medical videos like endoscopes, orthoscopic as a video water marking system.

\section{REFERENCES}

[1] P.Viswanthan,Member,IEEEandP.VenkataKrishna,Senior Member IEEE,"A Joint Fed Watermarking System Using Spatial Fusion For VerifyingThe Security Issues Of Tele-radiology",IEEE Journal of Biomedical and Health Informatics,Vol.18,No.3,May 2014.

[2] N.R.Brinta \& P.R.Bipin,"A Contourlet DomainWatermarkingAlgorithm", International Journal of Image Processing and Vision Sciences (IJIPVS) ISSN(Print): 2278 - 1110, Vol-1 Iss-3,4,2012.

[3] Ramin Eslami and Hayder Radha,"Wavelet-Based Contourlet Transform Andits Application To Image Coding",ECE Department, Michigan StateUniversity, East Lansing, MI 48824, USA

[4] charlesvanloan,"Using the Hessenberg Decomposition in Controltheory",Department ComputerScience, cornelluniversity,Ithaca,NewYork14853,U.S.A

[5] Hatem Ltaief, Jakub Kurzak, and Jack Dongarra,"ParallelBlockHessenbergReductionusingAlgorithms- By-Tiles for Multicore Architectures Revisited", LAPACK Working Note \#208.

[6] The Math Works, Inc., Getting Started with MATLAB. http://www.mathworks.com/access/helpdesk/help/t echdoc/learn_matlab/learn_matlab.sht ml 